\title{
Anomalous orientation effects in the Bourdon illusion
}

\author{
I. M. VERSTIJNEN and CEES VAN LEEUWEN \\ University of Amsterdam, Amsterdam, The Netherlands
}

\begin{abstract}
All current explanations of the Bourdon illusion imply that the illusion persists under mirror imaging over the vertical or horizontal axes. We tested this hypothesis in three experiments. For orientations of the standard Bourdon figure of about $135^{\circ}$, the illusion shows a dip, which is unexplainable in terms of current theories.
\end{abstract}

A configuration of two slender triangles that meet each other at their apexes (Figure 1) is known to produce the Bourdon illusion (Bourdon, 1902)-that is, the straight edge $A B C$ seems to bend inward, toward the opposite oblique edge EBD. Besides the solid variety of the figure, outline versions also have been studied (Wenderoth \& O'Connor, 1987). Day, Mitchell, and Stecher (1990) showed that the illusion in the solid variety occurs also with right angle and parallel edge figures. Our investigation will be restricted to the traditional, straight edge solid figures.

The magnitude of the Bourdon illusion has been found to depend on the orientation of the figure. Figure 2 shows illusion size as a function of orientation, according to Rozvany and Day (1980). The orientation dependence is shown to be an oblique effect (Appelle, 1972); the illusion is minimal at horizontal $\left(0^{\circ}\right)$ or vertical $\left(90^{\circ}\right)$ orientations and maximal at oblique ones (orientations between $0^{\circ}$ and $90^{\circ}$ ). The oblique effect is due to the relative sparsity of oblique edge detectors. Rozvany and Day's observations were restricted to figures between the horizontal and vertical orientation (Quadrant I). Walker and Shank (1987) observed the figures between mirrored horizontal $\left(180^{\circ}\right)$ and mirrored vertical $\left(270^{\circ}\right)$ orientations as well (Quadrant III). The authors showed that, if the figure is mirrored along the vertical axis, a mirror-imaged illusion size function can be obtained. They, however, left Quadrants II and IV to be investigated. The illusion size functions observed so far may lead to the prediction shown in Figure 3 for the Bourdon illusion size function in the four quadrants.

The present article reports three experiments, in which the illusion was studied in all four quadrants. This implied a possible partial replication of the earlier findings. To

The experiments reported in this arficle were part of a master's thesis by I.M.V. The authors thank Ronald Hamel for his useful suggestions, Lonneke Frie for testing the subjects of Quadrants III and IV in the first experiment, and Ingrid Christoffels for her contribution in performing the second experiment. Correspondence concerning this article should be sent to C. van Leeuwen, University of Amsterdam, Faculty of Psychology, Roetersstraat 15, 1018 WB Amsterdam, The Netherlands (e-mail: ceesvl@uvapsy.psy.uva.nl). minimize the possibility of procedure artifacts, different tasks were used in each of the experiments. A multiplechoice task was used in Experiment 1, a construction task in Experiment 2, and an adjustment task in Experiment 3.

\section{EXPERIMENT 1}

\section{Method}

Subjects. Twenty-two undergraduate psychology students- -4 male and 18 female-received course credit for participation. All had normal or corrected-to-normal vision.

Apparatus and Stimuli. Bourdon figures with arm length $\mathrm{AB} / \mathrm{BC}$ of $10 \mathrm{~cm}$ were printed in black on white, each in the center of a separate sheet of $29.6 \mathrm{~cm}$ (width) $\times 42.2 \mathrm{~cm}$ (height) paper. The inner apical angle CBD/ABE was $12.5^{\circ}$. These stimuli produced the strongest illusion in Rozvany and Day (1980).

A total of 16 different orientations of the Bourdon figure were used: $0^{\circ}$ (horizontal orientation); Quadrant I, $22.5^{\circ}, 45^{\circ}$, and $67.5^{\circ} ; 90^{\circ}$ (vertical orientation); Quadrant II, $112.5^{\circ}, 135^{\circ}$, and $157.5^{\circ} ; 180^{\circ}$ (mirrored horizontal orientation); Quadrant III, $202.5^{\circ}, 235^{\circ}$, and $257.5^{\circ} ; 270^{\circ}$ (mirrored vertical orientation); Quadrant IV, $292.5^{\circ}, 315^{\circ}$, and $337.5^{\circ}$.

For each orientation, there were 11 versions of the Bourdon figure. Across these alternative versions, the bend of edge $\mathrm{ABC}$ ranged from $-5^{\circ}$ to $+5^{\circ}$, in steps of $1^{\circ}$. A negative value implies bend toward EBD, a positive one away from EBD. The 11 versions were presented in a ring folder that was attached at eye level to the wall of the experiment room. Viewing distance was kept at $40 \mathrm{~cm}$.

Procedure. Alternative sets of figures were randomly assigned to the subjects. Twelve subjects ( 2 males and 10 females) received the set that consisted of the following orientations: horizontal, Quadrant I, vertical, and Quadrant II (Quadrant I--II group). Ten subjects ( 2 males and $8 \mathrm{fe}-$ males) received figures in these orientations: mirrored horizontal, Quadrant III, mirrored vertical, and Quadrant IV (Quadrant III-IV group). By presenting alternative sets of figures to different groups of subjects, confusion between the left side (Quadrant I-II) and the right side (Quadrant III-IV) was avoided.

The subjects were instructed to stand upright, facing the wall to which the ring folder was attached. They were asked to turn freely the pages of the ring folder, in order to examine the 11 alternative versions of a given orientation of the Bourdon figure. Binocular viewing was used. No time limits were imposed. The 11 versions were randomly ordered in the ring folder. The subjects were instructed to select the one perceived as straight. This was done three times for each orientation in the set in random order and lasted approximately $90 \mathrm{~min}$.

\section{Results and Discussion}

Two types of analyses were performed. The first one tested for the orientation dependence of the illusion. Sep- 


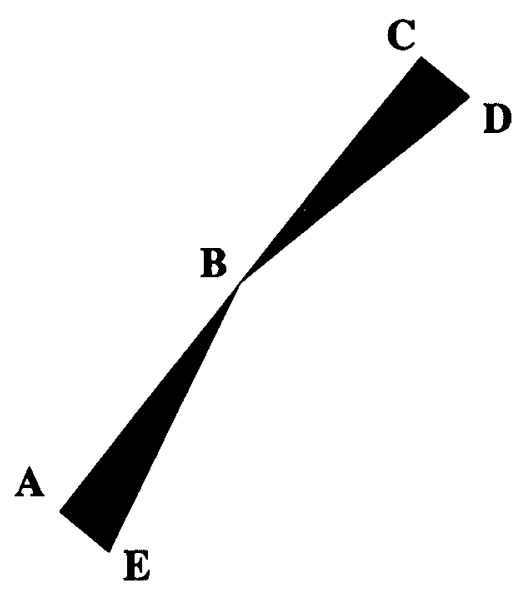

Figure 1. Bourdon figure. The straight line ABC seems bent toward the opposite oblique edge EBD.

arate one-factor analyses of variance (ANOVAs) were performed for observations within each quadrant. The orientation factor had five levels in Quadrants I and III and four levels in Quadrants II and IV, because the $0^{\circ}$ orientation was included with Quadrant $\mathrm{I}$, the $90^{\circ}$ orientation with Quadrants I and II, the $180^{\circ}$ orientation with Quadrant II, and the $270^{\circ}$ orientation with Quadrants III and IV. Orientation dependence was obtained for Quadrant I $[F(4,44)=20.395, p<.001]$, for Quadrant III $[F(4,36)=$ $27.763, p<.001]$, and for Quadrant IV $[F(3,27)=18.013$, $p<.001]$, but not for Quadrant II $[F(3,33)=1.754, p=$ $.175]$. Figure 3 shows that the effect of orientation in Quadrants I, III, and IV is an oblique effect: The illusion is maximal if the Bourdon figure is presented in an oblique orientation. The oblique effects in Quadrants I and III replicate the findings observed earlier (Walker \& Shank, 1987). The effect in Quadrant IV is in accordance with the prediction. The absence of orientation dependence in Quadrant II, however, violates the predicted oblique effect for this quadrant.

The absence of the oblique effect in Quadrant II may be a consequence of a reduction in the illusion for that quadrant. The second type of analysis was performed in order to compare the size of the illusion between the quadrants. The scores for orientations on opposite sides of the vertical axis were pairwise compared using $t$ tests. Between Quadrants III and IV, only the orientations $205.5^{\circ}$ versus $337.5^{\circ}$ differ in illusion $[t(9)=2.469, p=.036]$. Quadrants III and IV show a symmetry along the vertical axis (Figure 3). The only observed divergence from symmetry is a reduction in the size of the illusion for the orientation closest to Quadrant II. A clear divergence from symmetry, however, is observed in Figure 3 between Quadrants I and II. Significant differences were obtained for all scores: $22.5^{\circ}$ versus $157.5^{\circ}[t(11)=3.981, p=.002]$, $45^{\circ}$ versus $135^{\circ}[t(11)=4.307, p=.001]$, and $67.5^{\circ}$ versus $112.5^{\circ}[t(11)=7.433, p<.001]$. The illusion is sub- stantially reduced in Quadrant II and does not differ from zero $[t(11)=0.725, p=.242]$.

\section{EXPERIMENT 2}

\section{Method}

Subjects. Fifty-two undergraduate psychology students -50 female and 2 male-received course credit for participation. Their mean age was 20.96 years. All had normal or corrected-to-normal vision.

Apparatus and Stimuli. Eight sheet-iron $50 \times 50 \mathrm{~cm}$ square plates covered with white paper served as the background for a Bourdon figure. One black plastic triangle was fixed with its apex in the exact middle of each plate, protruding $1 \mathrm{~mm}$ from the surface. A second triangle of the same material was used to form the Bourdon figure. This triangle could be placed freely onto the plate by the subject. For this purpose, it had three little flat magnets glued to its back. The magnets were $1 \mathrm{~mm}$ thick. Both triangles were of the same size as those in the previous experiment.

Each plate was attached to the wall of the experiment room at eye level and was used for four orientations, by rotating it by $0^{\circ}, 90^{\circ}, 180^{\circ}$, or $270^{\circ}$. For example, a plate with triangle BCD (see Figure 1) under an orientation of $22.5^{\circ}$ will become an orientation of $112.5^{\circ}$ by $90^{\circ}$ of counterclockwise rotation. Hence, all 16 possible orientations of the Bourdon configuration from Experiment 1 were covered using eight plates.

Procedure. As in the previous experiment, figures from Quadrants I-II were presented to half of the subjects, and Quadrants III-IV to the others. On each trial, the subjects were standing in front of the wall to which the plate with one triangle in a fixed position was attached. The subjects stood approximately $40 \mathrm{~cm}$ from the wall and were told to keep their heads straight up. Half of the subjects received their trials with a

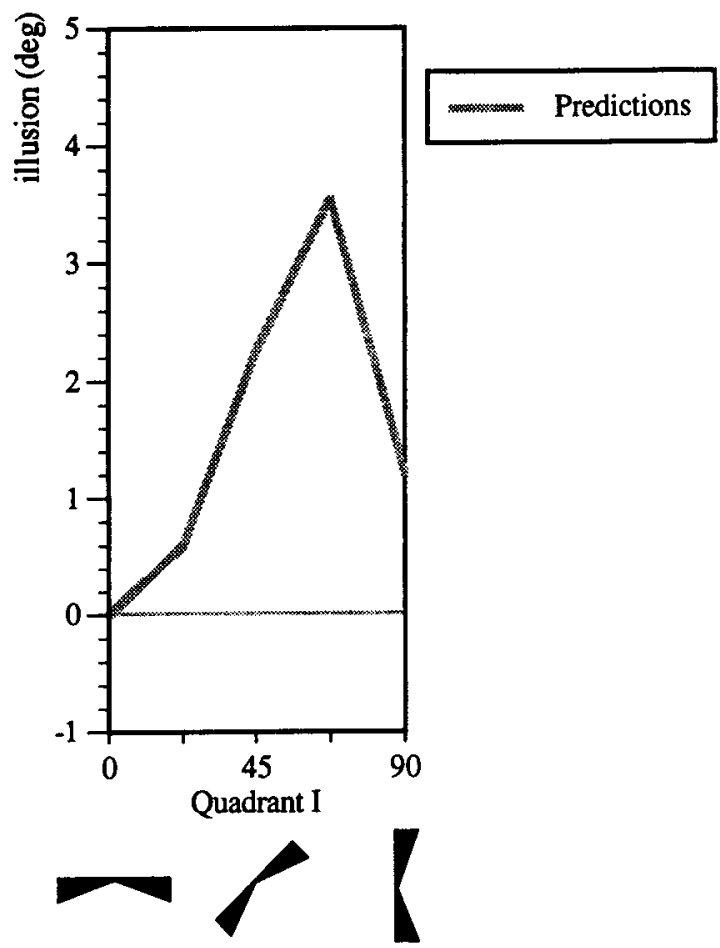

orientation of the Bourdon figure (deg)

Figure 2. Bourdon illusion as a function of the figure orientation (adapted from Rozvany \& Day, 1980). 


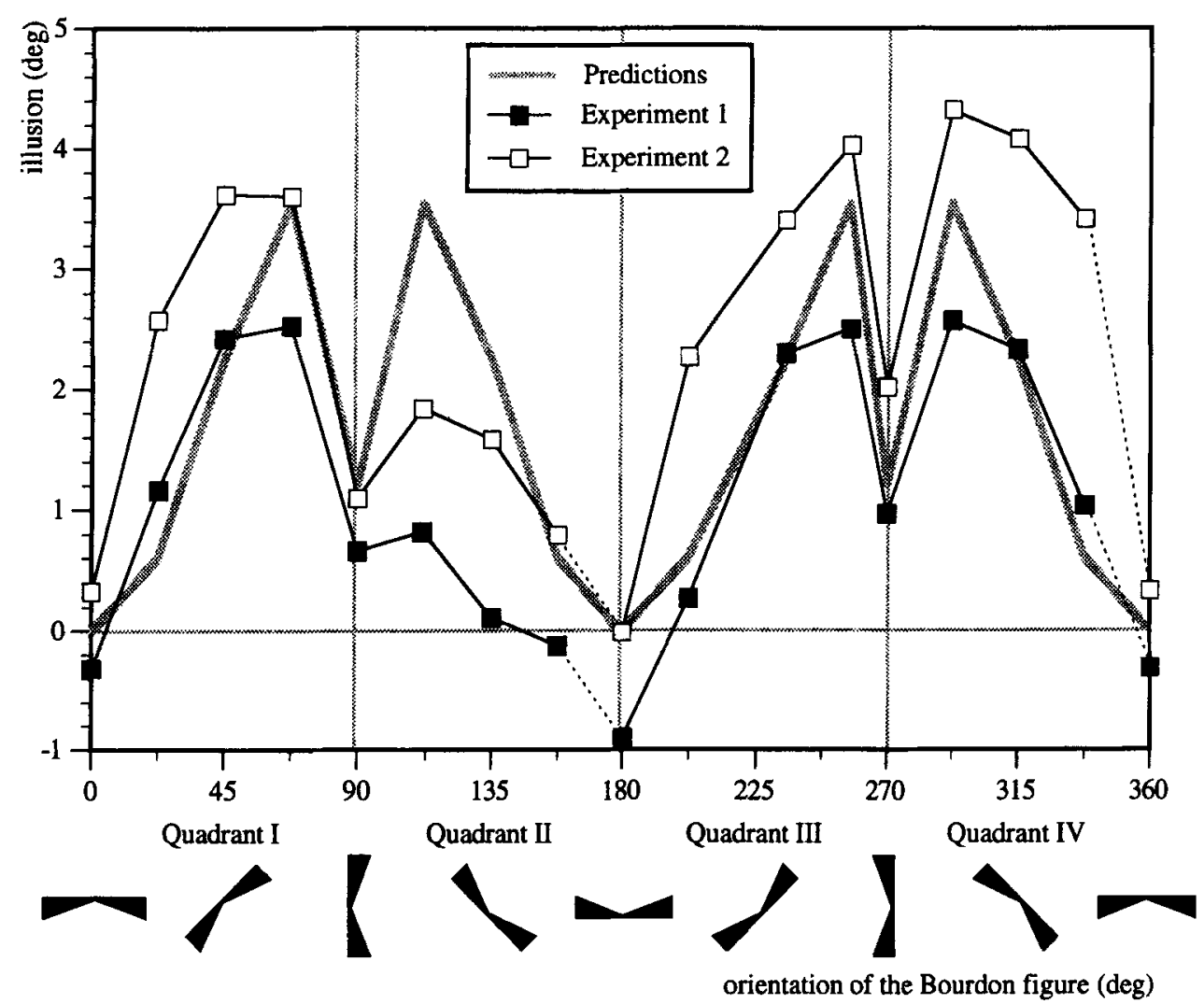

Figure 3. The predicted orientation dependence of the Bourdon illusion, and the illusions as found in Experiment 1 and Experiment 2. In both experiments, the illusion shows an unpredicted dip in Quadrant II.

fixed upper triangle, and the others received a fixed lower triangle. The subjects were handed the second triangle. They were instructed to place it on the plate, aligning it with the fixed triangle in such a manner that line $A B C$ in Figure 1 formed a straight line. The subjects were allowed to make adjustments by moving their triangle on the plate, without time restrictions. Afterwards, the deviation from the straight line was measured with a transparent overlay, while the subject was performing the next trial. The subjects got four series of trials. Each series contained eight orientations of the Bourdon configuration, as in Experiment 1, in random order, with the restriction that the same plate was not used on two consecutive trials.

\section{Results and Discussion}

No differences were established between upper and lower triangle alignment conditions. These scores, therefore, were pooled, yielding 26 subjects for Quadrant I-II as well as for Quadrant III-IV. Initially, subjects showed evidence of confusion between line BD and BC. The data from the first series were therefore dismissed as practice trials. The means of the second through fourth series constitute the subjects' scores.

Two types of analyses were performed on these scores, as in Experiment 1. In the first analysis, which tested for the orientation dependence of the illusion, separate onefactor ANOVAs were performed for observations within each quadrant. Orientation dependence was obtained for
Quadrant I $[F(4,100)=50.507, p<.001]$, for Quadrant III $[F(4,100)=29.869, p<.001]$, for Quadrant IV $[F(3,75)=$ $9.127, p<.001]$, but also for Quadrant II $[F(3,75)=3.541$, $p=.019]$. As shown in Figure 3, the orientation effects in Quadrants I, III, and IV replicate the oblique effect. The orientation effect in Quadrant II is not an oblique effect: to be identified as an oblique effect the illusion obtained for oblique orientations within this quadrant has to differ from both $90^{\circ}$ and $180^{\circ}$. For oblique orientations, a significant difference could only be established from $180^{\circ}$ $[t(50)=2.992, p=.004]$ but not from $90^{\circ}[t(25)=1.172$, $p=.252]$.

In the second analysis, which compared the illusion between the quadrants, scores for opposite sides of the vertical axis were pairwise compared with $t$ tests. As in the first experiment, for Quadrants III-IV only orientations $205.5^{\circ}$ versus $337.5^{\circ}$ differ in illusion $[t(25)=2.203$, $p=.037]$. The illusion differs significantly between Quadrants I and II for all orientations: $22.5^{\circ}$ versus $157.5^{\circ}$ $[t(25)=4.871, p<.001], 45^{\circ}$ versus $135^{\circ}[t(25)=5.555$, $p<.001]$, and $67.5^{\circ}$ versus $112.5^{\circ}[t(25)=5.144, p<$ $.001]$. As is shown in Figure 3, the reduction in illusion observed in the present experiment is identical to that of Experiment 1. 


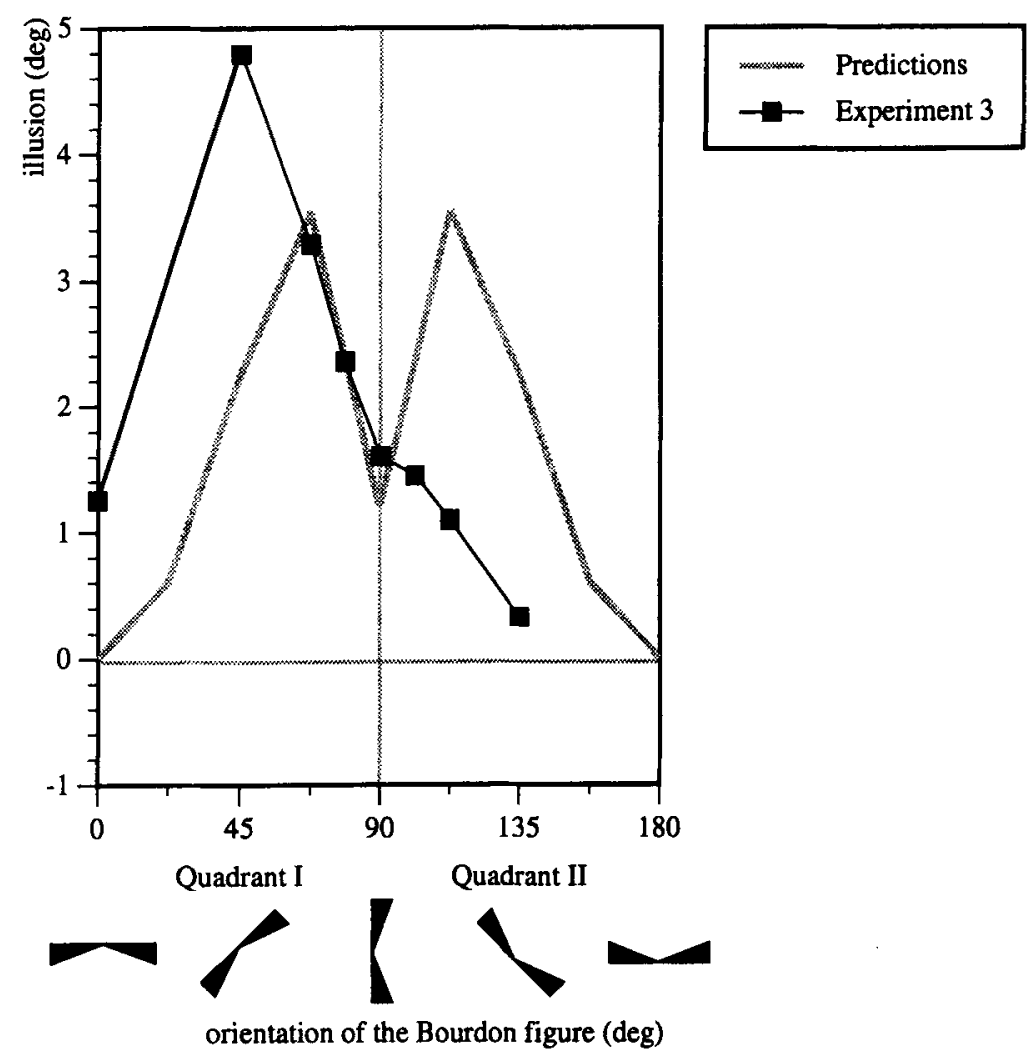

Figure 4. The predicted orientation dependence of the Bourdon illusion and the illusion as found in Experiment 3. Again the illusion shows an unpredicted dip in Quadrant II.

\section{EXPERIMENT 3}

\section{Method}

Subjects. Four undergraduate psychology students, 2 male and 2 female, received course credit for participation. All had normal or corrected-to-normal vision.

Apparatus and Stimuli. A viewing box was constructed whose cylinder-shaped interior minimized orientation cues other than those from the Bourdon figure itself. The lower triangle of the Bourdon figure could be set in a prearranged orientation by the experimenter by means of a handle on the outside of the box. Eight different orientations were used: $0^{\circ}, 45^{\circ}, 67.5^{\circ}, 78.75^{\circ}, 90^{\circ}, 101.25^{\circ}, 112.5^{\circ}$, or $135^{\circ}$. The upper triangle could be adjusted by the subject by turning a wheel on the front panel of the box. A pointer on a protractor scale indicated the size of the illusion to the experimenter. The subject's head was fixed in order to exclude head tilt.

The subjects viewed black Bourdon figures binocularily against a white background, with an illumination of approximately 20 lux from a fixed distance of $48 \mathrm{~cm}$. The top triangle in the illusion was located at a distance of $49.5 \mathrm{~cm}$ from subjects' viewing point. Because of the unfortunate construction, the closest location of the bottom triangle to the top triangle was at $57.5 \mathrm{~cm}$ distance. Both triangles subtended the same $20^{\circ}$ of visual angle. The acute angle of both triangles again was $12.5^{\circ}$

Procedure. The subjects were instructed to turn the wheel in order to align the upper triangle of the Bourdon figure to the one set by the experimenter. On each trial, the experimenter set the lower triangle at one of the eight prearranged orientations. Eight randomized series of eight orientations were presented. The subjects were given unrestricted time to complete a trial.

\section{Results and Discussion}

Because a long practice session preceded the trials, the average from all eight series could be used. The significant $[F(4,12)=6.613, p=.0047]$ orientation dependence for Quadrant I is shown in Figure 4 to be an oblique effect. A marginally significant $[F(3,9)=4.261, p=$ .0394 ] orientation effect is observed in Quadrant II, which, however, cannot be interpreted as an oblique effect. In spite of the shortcomings of the apparatus, these observations essentially duplicate the results of Experiments 1 and 2.

\section{GENERAL DISCUSSION}

Four different explanations for the Bourdon illusion have been proposed. Walker and Shank (1988) started from the distribution of orientation-specific edge detectors. These authors suggested that the illusion results from lateral inhibition from the edges in combination with neural excitation from the solid in-between. The net effect is a mutual attraction of the two neighboring edges ( $\mathrm{AB}$ and $\mathrm{EB}$ or $\mathrm{BC}$ and $\mathrm{BE}$ ). Wenderoth, O'Connor, and Johnson (1986) proposed an alternative explanation, in which the reduced salience of the straightness and collinearity cues of a solid Bourdon (as opposed to an outline Bourdon) are held responsible for the illusion. A third explanation (Walker \& Shank, 1988) attributed the Bourdon effect to a major confusion between the straight and obtuse edges of the triangles within oblique orientations of the Bourdon figure. According to a fourth proposal by Day (1989, 1990), 
the illusion can be accounted for in terms of a conflict between the respective orientation cues for the edge $(\mathrm{ABC})$ and those of the two slender triangles that make up the figure, as represented by their bisectors.

All four explanations predicted that the illusion would occur on all four quadrants. Moreover, in all quadrants an oblique effect would be expected. The reduction of the illusion and the absence of an oblique effect in Quadrant II observed in all three of our experiments cannot be explained by any of these theories.

It is unclear which assumption could be added to these theories in order to explain the effects. The lines to be judged in Quadrant II are left-skewed. A relevant observation, therefore, might seem to be Rochlin's (1955) finding that subjects are more accurate in detecting the orientation of left-skewed lines. However, this would lead us wrongly to expect as well a reduction in the illusion in Quadrant IV, in which the line to be judged is also left-skewed.

A tentative explanation for why the effect occurs only in Quadrant II but not in Quadrant IV could reside in the additional assumption that the figure is interpreted relative to a virtual horizon (Rock, 1974). Assuming that the base of the Bourdon figure is placed at this horizon, this would result in a virtual angle with line $\mathrm{ABC}$ and the internal horizontal axis. In Quadrants I and IV, the establishment of this virtual angle is hindered by the fact that the Bourdon figure itself is lying between line $\mathrm{ABC}$ and the horizon. In contrast, in Quadrants II and III, no figure disturbs the angle. This advantage for Quadrants II and III, in combination with Rochlin's (1955) finding of an advantage for left-skewed linesthat is, Quadrants II and IV-would add up to be a major advantage for Quadrant II.

The fact that the illusion grows, even in Quadrant II, the more distant the figure is from the horizontal may support this suggestion. The minor reduction in the illusion that occurs for the $202.5^{\circ}$ orientation in Quadrant III, which is closest to the horizon, may also be interpreted in support of this assumption. The virtual horizon hypothesis would also lead us to predict a smaller illusion for $180^{\circ}$ than for $0^{\circ}$ orientations. The difference, although in the predicted direction, is nonsignificant, but this may be due to the fact that the illusion is already minimal for these orientations.

It is important in this context that the illusions also occur when the two thin triangles are placed side by side with their edges parallel and at an angle with their edges at right angles (Wenderoth, 1991). These arrangements result in illusions of nonparallelness and nonright angularity, respectively (Day et al., 1990). It may be worthwhile, therefore, to investigate whether the obtained quadrant effect is specific to the conventional apex-to-apex arrangement of the two triangles or whether it occurs also with these other arrangements. Alternative suggestions would be to study the quadrant effect in haptic space (Day, 1990) or in subjective contour versions (Walker \& Shank, 1987). These issues are open to further investigation.

\section{REFERENCES}

APPELLE, S. (1972). Perception and discrimination as a function of stimulus orientation: The "oblique effect" in man and animals. Psychological Bulletin, 78, 266-278.

Bourdon, B. (1902). La perception visuelle de l'espace [The visual perception of space]. Paris: Reinwald.

DAY, R. H. (1989). Natural and artificial cues, perceptual compromise and the basis of veridical and illusory perception. In D. Vickers \& P. L. Smith (Eds.), Human information processing: Mechanisms and models (pp. 107-129). London: Elsevier.

DAY, R. H. (1990). The Bourdon illusion in haptic space. Perception \& Psychophysics, 47, 400-404.

Day, R. H., Mitchell, P., \& Stecher, E. J. (1990). The Bourdon illusion occurs with straight-, right-angle-, and parallel-edge figures. Perception \& Psychophysics, 48, 375-381.

Rochlin, A. M. (1955). The effect of tilt on the visual perception of parallelness. American Journal of Psychology, 68, 223-236.

Rock, I. (1974). The perception of disoriented figures. Scientific American, 230, 78-85.

Rozvany, G. I., \& DaY, R. H. (1980). Determinants of the Bourdon effect. Perception \& Psychophysics, 28, 39-44.

WaLKER, J. T., \& ShaNK, M. D. (1987). The Bourdon illusion in subjective contours. Perception \& Psychophysics, 42, 15-24.

WALKER, J. T., \& SHANK, M. D. (1988). Real and subjective lines and edges in the Bourdon illusion. Perception \& Psychophysics, 43, 475-484.

WENDEROTH, P. (1991). The basis of the Bourdon illusion. Perception \& Psychophysics, 49, 393-397.

WENDEROTH, P., \& O'CONNOR, T. (1987). Outline- and solid-angle illusions have different determinants. Perception \& Psychophysics, 41, 45-52.

WENDEROTh, P., O'CONNOR, T., \& Johnson, M. (1986). The tilt illusion as a function of the relative and absolute lengths of test and inducing lines. Perception \& Psychophysics, 39, 339-345.

(Manuscript received May 2, 1994;

revision accepted for publication September $29,1997$. 